Original article (short paper)

\title{
Acute effects of maximal isometric muscle action of the elbow extensors on contralateral dynamic task of the elbow flexors: a pilot study
}

\author{
Cauê V La Scala Teixeira \\ Universidade Federal de São Paulo, UNIFESP, Santos, SP, Brazil \\ Faculdade Praia Grande, FPG, Praia Grande, SP,Brazil \\ Diego R Cortes \\ Universidade Metropolitana de Santos, UNIMES, Santos, SP, Brazil \\ Alexandre L Evangelista \\ Universidade Nove de Julho, UNINOVE, São Paulo, SP, Brazil \\ Luis T Polito \\ Danilo S Bocalini \\ Universidade São Judas Tadeu, São Paulo, SP, Brazil \\ Pablo B. Costa \\ California State University, Fullerton, USA \\ Roberto Simão \\ Universidade Federal do Rio de Janeiro, UFRJ, Rio de Janeiro, RJ, Brazil \\ Paulo H Marchetti \\ California State University Northridge, Northridge, CA, USA
}

\begin{abstract}
Aim: the aim was to investigate the influence of a maximal isometric muscle action of the elbow extensors on the contralateral dynamic task of the elbow flexors. Methods: Seventeen recreationally trained men $(23.3 \pm 4.9 \mathrm{yrs}$, BMI: $\left.24.8 \pm 2.2 \mathrm{Kg} / \mathrm{m}^{2}\right)$ underwent two randomized different testing sessions separated by one week. In the control session (CON) all subjects performed a maximum number of repetitions test (RMs) at 75\%1RM using the right elbow flexors. The experimental session (EXP) was similar to the CON; however, all subjects were instructed to perform RMs at 75\%1RM by using the right elbow flexors and maintaining the maximal voluntary contraction of the left elbow extensors during the test. RMs, rating of perceived exertion (RPE), and training volume (TV) were measured and compared between sessions. Results: The EXP showed a significant $10.4 \%$ increase on the RMs $(13.8$ vs. $12.5, p<$ $0.001, d=0.44)$ and $12.1 \%$ increase in TV (238.0 vs. $212.4 \mathrm{~kg}, p<0.001, d=0.43)$ than CON. No differences were observed for RPE between sessions. Conclusion: The maximum voluntary contraction of the left elbow extensors increased the RMs of the contralateral elbow flexors, reflecting a higher TV, and no differences in the RPE. Our results suggest that the investigated method may be a viable and practical alternative to increase the acute strength performance of elbow flexors when using submaximal loads.
\end{abstract}

Keywords: resistance training, reciprocal activation, strength, physical performance, remote voluntary contraction

\section{Introduction}

Optimal prescription of resistance training programs relies on proper organization of training variables such as frequency, intensity, volume, rest intervals, velocity, choice and order of exercise, and periodization ${ }^{1,2}$. Previous research has demonstrated the importance of varying training methods to provide increasing and consistent results ${ }^{3-5}$. One of these methods consists of performing an exercise that requires simultaneous actions of contralateral muscles that perform opposite movements, for example, the right elbow flexors and the left elbow extensors ${ }^{6}$. Interactions between the right and left sides of the body are known to produce various effects upon human performance, and it is well known that the muscle action of one side of the body is mainly controlled by the contralateral cerebral hemisphere ${ }^{7}$. The two cerebral hemispheres are connected by commissural nerve fibers, and the most conspicuous of these connections is the corpus callosum. Weineck ${ }^{8}$ suggests muscle tension and consequently acute performance are enhanced when the contralateral opposite muscle is contracted at the same time as a dynamic task is performed by the exercise primary mover, that is, the performance in a right elbow flexor task is increased if, simultaneously, the left elbow extensor is contracted.

The increase of strength performance of muscles via the contraction of remote muscles to the prime mover is a phenomenon that has been described as remote voluntary contractions ${ }^{9,10}$ or concurrent activation potentiation (CAP) ${ }^{11}$. This phenomenon has been widely investigated; however, most studies investigating the effects of remote voluntary contraction have examined the influence of upper limb contraction on the performance of lower limb tasks and vice versa ${ }^{9,12}$, as well as strategies such as jaw clenching and Valsava maneuver ${ }^{13}$. In these situations, the observed increase in performance is due to hypotheses such as theory of motor overflow (TMO) ${ }^{9,14}$ and the summation of proprioceptive inputs (SPI $)^{10}$. However, Weineck ${ }^{8}$ has suggested that the increase in performance by simultaneous contraction of contralateral muscles performing opposite movements might be explained (at least in part) by the contralateral coactivation 
phenomenon, although, there is a gap in the literature about the effects of contralateral coactivation on acute performance.

Ohtsuki ${ }^{7}$ demonstrated that during the voluntary muscle action of the right triceps brachial, the biceps brachial of opposite limb was involuntarily reciprocally activated. Although this was not the objective of the Ohtsuki's paper, the reciprocal activation is identified through an example of recorded traces in the EMG analysis presented in Figure 2 of the mentioned article. Thus, theoretically, voluntary activation of the contralateral biceps brachial might excite the opposite triceps brachial due to the contralateral coactivation phenomenon, and this can increase the performance of the latter. Therefore, the aim of this study was to investigate the immediate influence of the maximal isometric muscle action of the elbow extensors during the contralateral dynamic task of the elbow flexors. We hypothesized that the voluntary left elbow extensor activation may enhance the acute performance of the right elbow flexor.

\section{Methods}

\section{Experimental Approach to the Problem}

In order to examine the effects of contralateral voluntary muscle action of the elbow extensor on acute performance and perceived exertion of an opposite elbow flexor task, resistance-trained men visited the laboratory three times separated by one week. On the first visit, subjects performed a one repetition maximum test (1RM test) of the right elbow flexion exercise on a Scott bench equipment. The following randomized sessions included control and experimental conditions. In the control session, subjects performed a maximum repetitions test of the right elbow flexion exercise at $75 \% 1 \mathrm{RM}$. The experimental session was similar; however, the subjects maintained a voluntary maximum isometric muscle action of the left elbow extensors during the test. The maximum number of repetitions and rating of perceived exertion were measured and compared between conditions.

\section{Subjects}

Seventeen young, resistance-trained men, without previous disease diagnosis, right-arm dominant based on their preferred arm to write (age \pm SD: $23.3 \pm 4.9$ years, height: $173.0 \pm 5.2 \mathrm{~cm}$, total body mass: $74.2 \pm 7.35 \mathrm{Kg}$, BMI: $24.8 \pm 2.2 \mathrm{Kg} / \mathrm{m}^{2}$, resistance training experience: $3.3 \pm 2.3$ years, right elbow flexion 1RM: $22.6 \pm 4.6 \mathrm{~kg}$ ) volunteered to participate in the current study. Exclusion criteria included current chronic pain, recent surgery ( $<1$ year), recent hospitalization, any chronic disease, cognitive impairment or progressive conditions and with debilitating inability to exercise, recent bone fractures $(<1$ year), and any other medical contraindications for training. All participants had medical examinations and completed questionnaires regarding medical history, and protocols were performed in accordance with the ethical standards of the Helsinki Declaration. This study was approved by the University (FEFISA Educational Center, $\mathrm{n}^{\circ}$ 1.136.301, 02/jul/2015) research ethics committee and all subjects read and signed an informed consent document.

\section{Experimental design}

Volunteers attended three sessions in the laboratory, and they refrained from performing any upper body exercise other than activities of daily living for at least one week between sessions. During the first session, all subjects performed a 5-min cycle warm-up at 70rpm and a familiarization with all experimental protocols. Then, all subjects performed a 1RM test of the right elbow flexion exercise on a preacher bench. The following experimental sessions were randomized for each subject, and a one week interval was adopted among sessions (1RM test, control session, and experimental session). In the control session, after a specific warm-up ( 1 set of 10 repetitions with $20 \%$ of $1 \mathrm{RM})$, all subjects performed a maximum number of repetitions test at $75 \%$ of $1 \mathrm{RM}$ with the right elbow flexors on a preacher bench equipment and free cadence. The experimental session was similar to the control session; however, subjects were instructed to perform a maximum number of repetitions test at $75 \%$ of $1 \mathrm{RM}$ with the right elbow flexors on a preacher bench equipment while maintaining the maximal voluntary contraction of the left elbow extensors during the test, keeping the left arm parallel to the trunk (figure 1). Warm up and maximum repetition tests were performed at $20 \% 1 \mathrm{RM}$ and $75 \% 1 \mathrm{RM}$, respectively, since these intensities are commonly used in workout routines and are in accordance to training recommendations for muscle hypertrophy ${ }^{15}$.

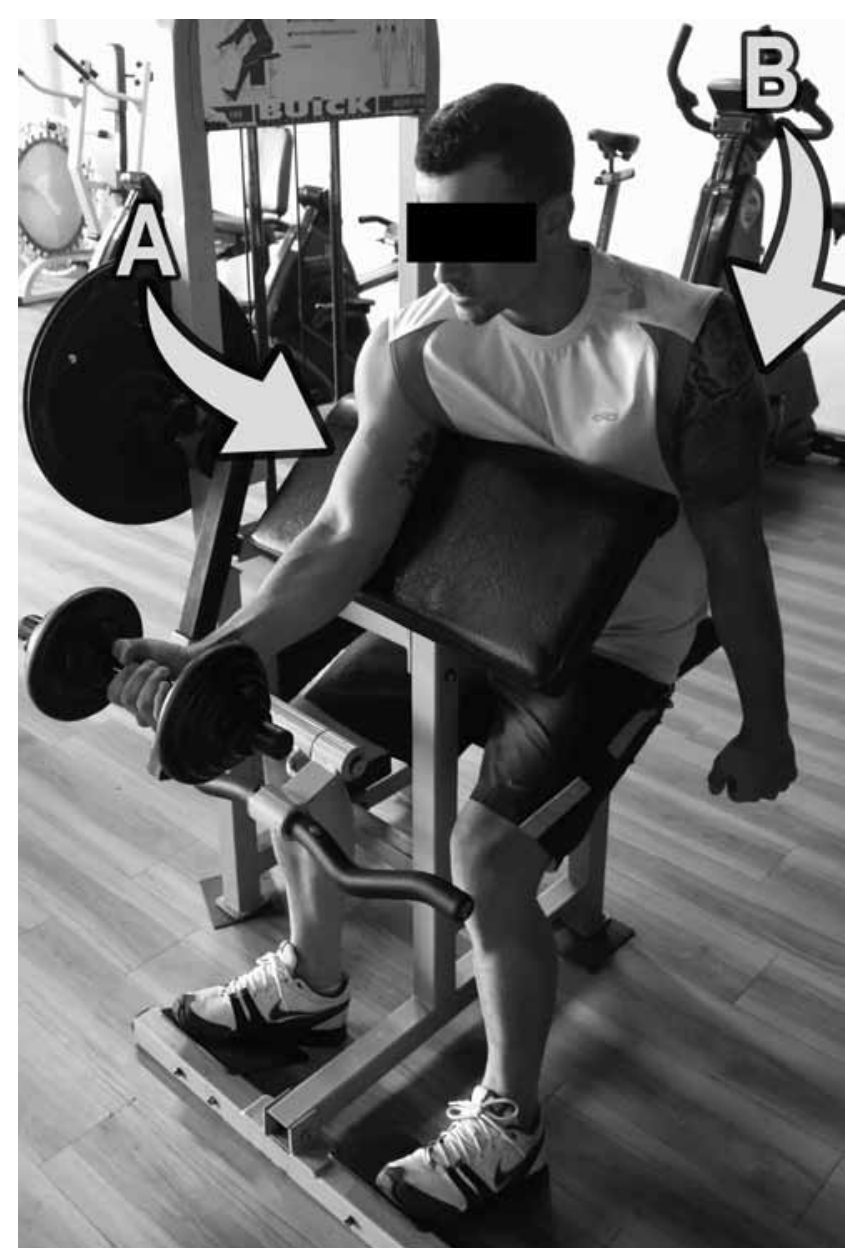

Figure 1. Experimental condition: (A) dynamic task of the right elbow flexor; (B) maximum isometric contraction of the left elbow extensor. 
At the end of both sessions (control and experimental), the maximum number of repetitions, correctly performed until voluntary fatigue, and the rating of perceived exertion (RPE) were assessed. In addition, the training volume (TV) was calculated for each session by multiplying the number of sets by total number of repetitions by the lifted weight $(\mathrm{kg})$ according to previous study ${ }^{16}$. All measures were performed at approximately the same period of day (morning), between 9:00 AM and 12:00 $\mathrm{PM}$, and by the same researcher.

\section{Maximum repetition testing (1RM)}

All subjects performed a 1RM test of the right elbow flexion exercise on the preacher bench. Subjects received standard instructions regarding the technique. Exercise execution was monitored and corrected when necessary, ensuring no stopping between eccentric and concentric phases for each test. The cadence during the test was free. For a successful repetition, the maximum range of motion was predefined for each exercise. After a previous warm-up (10 repetitions of the specific exercise with $20 \%$ of estimated 1RM load), all subjects started a maximum of five 1RM attempts with 5-minute rest intervals between trials ${ }^{17}$. In order to avoid possible errors in the $1 \mathrm{RM}$ test, the following strategies were adopted: all subjects received standard instructions regarding the general routine of data assessment and the exercise technique before testing; the exercise technique of subjects was monitored and corrected by an experienced personal trainer.

\section{Rating of perceived exertion (RPE)}

An OMNI-RES RPE scale ${ }^{18}$ was used to assess effort during the conditions. Standard instructions and anchoring procedures were explained during the familiarization session. Subjects were asked to use a number on the scale to rate their overall effort for each condition. A rating of " 0 " was associated with no effort and a rating of " 10 " was associated with maximal effort and the most stressful exercise ever performed. Subjects were shown the scale immediately after each condition and asked: "How was your workout?".

\section{Statistical analysis}

Normality and homogeneity of variances were confirmed with the Shapiro-Wilk and Levene's tests, respectively. All data were expressed as mean \pm standard deviation. Paired samples t-test was used to compare differences between experimental and control conditions for all dependent variables (maximum number of repetitions at 75\% 1RM; TV; RPE). Cohen's formula for effect size $(d)$ was calculated, and the results were interpreted based on the following criteria: $<0.35$ trivial effect; $0.35-0.80$ small effect; $0.80-1.50$ moderate effect; and $>1.5$ large effect, for recreationally trained subjects ${ }^{19}$. An alpha level of 0.05 was used to determine statistical significance. All statistical analyses were performed using SPSS software (v 15.0; IBM, Armonk, NY, USA).

\section{Results}

The maximum number of repetitions at 75\% 1RM was significantly greater in the experimental condition than control (13.8 \pm 4 reps vs. $12.5 \pm 3$ reps, respectively; $p<0.001, d=$ 0.44 ) (Figure $2 \mathrm{~A}$ ). The TV was also significantly greater in the experimental condition than control $(238 \pm 59 \mathrm{~kg}$ vs. $212 \pm 59$ $\mathrm{kg}$, respectively; $p<0.001, d=0.43$ ) (Figure 2B). However, there was no significant difference for RPE between sessions (experimental: $7.4 \pm 1.0$ vs. control: $7.5 \pm 1.2, p=0.749, d=$ -0.08) (Figure 2C).
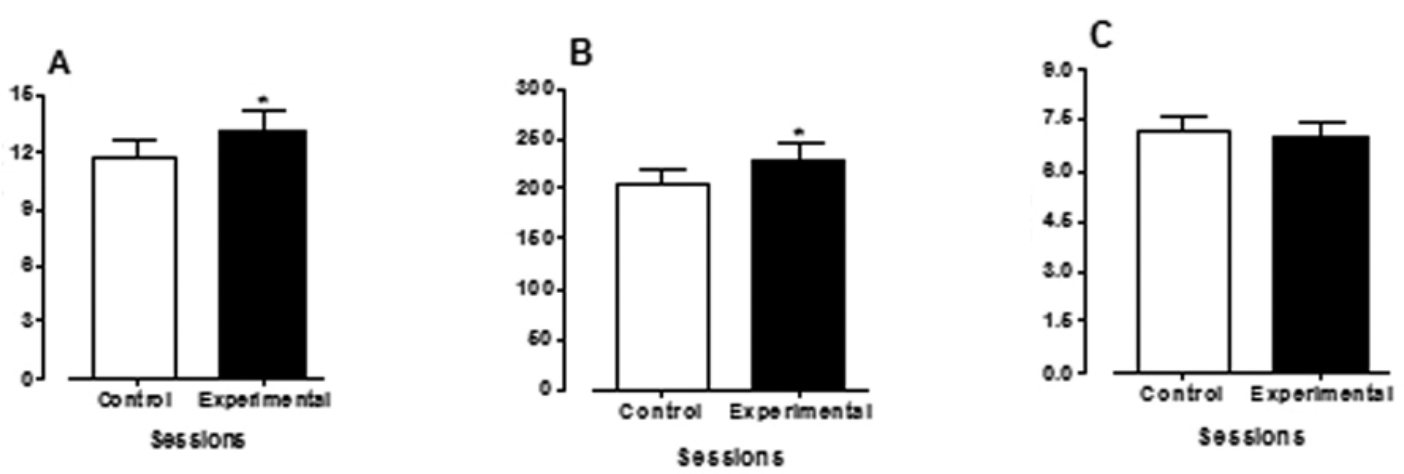

Figure 2. Means and standard deviation of the following dependent variables. A: maximum number of repetitions at 75\%1RM; B: training volume; C: RPE in both conditions (control and experimental); ${ }^{*}$ Significant differences between Experimental and Control $(p<0.001)$.

\section{Discussion}

The motivation for performing this research was based on the hypothesis that the performance would increase in the experimental condition, as suggested by Weineck ${ }^{8}$. This initial hypothesis was confirmed. The experimental condition showed a significant increase (10.4\%) on maximum repetitions performance than control condition, reflecting the significantly increase 
of TV by $12.1 \%$. These results suggest that the investigated method may be a viable and practical alternative to increase the acute strength performance of elbow flexors when using submaximal loads.

The maximum number of repetitions and TV are considered an important stimulus for morphological and functional adaptations because they imply greater levels of mechanical tension and metabolic stress ${ }^{15}$. The mechanical tension is the primary characteristic which leads to strength gains, whereas, the metabolic stress contributes to increasing the muscular endurance. The increase of mechanical tension by the greater number of repetitions with heavy weights (as noted in the present study) is a characteristic that may lead to higher gains in muscle mass ${ }^{3}$. Similarly, the metabolic stress plays an important role in hypertrophy ${ }^{20}$. Additionally, the TV has been suggested as a variable that directly influences the hypertrophic results. Therefore, training methods that elevate the TV tend to provide better responses on the increase of muscle mass ${ }^{21,22}$. A relevant effect observed in our study was that the increase in the maximum number of repetitions and the TV was not reflected in the RPE values. Thus, the method used in this study presented an option for situations in which an increase in performance is desired without increasing the perceived exertion.

One fact that might explain the increased performance of the contralateral flexor muscle group is the reciprocal activation of the contralateral elbow extensor muscle, as noted by Ohtsuki ${ }^{7}$. In that particular study, the recorded traces in the EMG analysis showed an increase in involuntary activation of biceps brachial in response to the voluntary isometric contraction of the contralateral triceps brachial. Thus, in the present study, the voluntary contraction of left elbow extensors has possibly increased the activation of the right elbow flexors (exercised muscles), raising the capacity of the weight lifted. Previous studies have shown non-local muscle activation (remote voluntary contractions) can promote excitatory signals between different cortical areas, resulting in facilitating the enhancement of the activation of other muscles ${ }^{23,24}$. In addition, the cross facilitation phenomenon is related to the propagation of neural signals between the left and right hemispheres in both brain level as in spinal level ${ }^{25}$.

Some researchers have demonstrated voluntary contraction of upper limb muscle may enhance the action of the contralateral homologous muscle $\mathrm{e}^{26,27}$, for example, voluntary contraction of right biceps brachial increase the action of left biceps. However, to the best of our knowledge, this is the first study investigating the effects on non-homologous muscle performance (e.g., biceps and triceps). In addition to cross facilitation, other hypotheses that may explain the results observed in this study are the theory of motor overflow (TMO) $)^{9,14}$ and the summation of proprioceptive inputs $(\mathrm{SPI})^{10}$. According to the TMO, when a portion of the brain is activated, other parts are also stimulated ${ }^{9}$, which demonstrates that when a cortical region is activated due to voluntary contraction, the same area in the opposite hemisphere may be activated ${ }^{14}$. Another theory to explain the effects of remote muscle contractions is summation of proprioceptive inputs to the motor cortex ${ }^{28}$. When additional remote muscles are activated there is an increase in the number of possible signals sent to the motor cortex. This response improves the chance for signals to overlap and affect other areas of the motor cortex ${ }^{10}$.

As this is a pilot study, some limitations must be noted, mainly related to the non-use of tools to evaluate the neural aspects. Therefore, future investigations should explore the method along with central and/or peripheral excitability techniques (e.g., electromyographic, transcranial magnetic stimulation, electroencephalography, Hoffmann's reflex) to examine whether the neurophysiological hypothesis raised at the present time can be confirmed.

\section{Conclusion}

In conclusion, the maximum voluntary contraction of the left elbow extensors increased the number of maximum repetitions in the contralateral right elbow flexors task, reflecting in a higher $\mathrm{TV}$, and no differences in RPE compared to a control condition. Future studies are needed to investigate the neurophysiological mechanisms responsible for the increase in performance with these methods of training, as well as longitudinal research is required to examine whether the acute increase showed in performance will reflect in greater chronic adaptations, mainly related to strength, muscular endurance, and hypertrophy.

\section{Practical applications}

Our results suggest that the investigated method may be a viable and practical alternative to increase the acute performance when using submaximal loads (e.g., 75\% 1RM). In addition, the acute increase in the number of maximum repetitions and total volume can lead to increased of mechanical tension and metabolic stress levels, suggesting that the method has great potential to be used in intensifying resistance training phases.

\section{References}

1. Baker JS, Mccormick MC, Robert A, Robergs, RA. Interaction among skeletal muscle metabolic energy systems during intense exercise. J Nutr Metab. 2010;ID 905612.

2. Foster C, Florhaug JA, Franklin J, Gottschall L, Hrovatin LA, Parker $\mathrm{S}$, et al. A new approach to monitoring exercise training. J Strength Cond Res. 2001; 15(1):109-15.

3. Keogh JWL, Wilson GJ, Weatherby RP. A cross-sectional comparison of different resistance training techniques in the bench press. J Strength Cond Res. 1999;13(3):247-58.

4. Maia MR, Willardson JM, Paz GA, Miranda H. Effects of different rest intervals between antagonist paired sets on repetition perfomance and muscle activation. J Strength Cond Res. 2014;28(9):2529-35.

5. Schoenfeld BJ. The use of specialized training techniques to maximize muscle hypertrophy. Strength Cond J 2011;33:60-5.

6. La Scala Teixeira CV, Guedes Júnior, DP. Functional ressitance training: extending the limits of traditional prescription [in Portuguese]. 2nd Ed. Sao Paulo, Phorte, 2016. 
7. Ohtsuki T. Decrease in human voluntary isometric arm strength induced by simultaneous bilateral exertion. Behav Brain Res. 1983;7(2):165-78.

8. Weineck J. Optimales training [in Deutsh]. 16nd Ed. Balingen, Spitta; Auflage, 2009.

9. Ebben WP, Leigh DH, Geiser CF The effect of remote voluntary contractions on knee extensor torque. Med Sci Sports Exerc. 2008;40(10):1805-9.

10. Cherry EA, Brown LE, Coburn JW, Noffal GJ. Effect of remote voluntary contractions on knee extensor torque and rate of velocity development. J Strength Cond Res. 2010;24(9):2564-9.

11. Ebben WP. A brief review of concurrent activation potentiation: theoretical and practical constructs. J Strength Cond Res 2006;20(4):985-1.

12. Tazoe T, Endoh T, Nakajima T, Sakamoto M, Komiyama T. Disinhibition of upper limb motor area by voluntary contraction of the lower limb muscle. Exp Brain Res. 2007;177(3):419-30.

13. Ebben WP, Petushek EJ, Fauth ML, Garceau LR. EMG analysis of concurrent activation potentiation. Med Sci Sports Exerc. 2010;42(3):556-62.

14. Hoy KE, Fitzgerald PB, Bradshaw JL, Armatas CA, GeorgiouKaristianis N. Investigating the cortical origins of motor overflow. Brain Res Rev. 2004;46(3):315-27.

15. Ratamess NA, Alvar BA, Evetoch TK, Housh TJ, Kibler WB, Kraemer WJ, et al. American College of Sports Medicine position stand: progression models in resistance training for healthy adults. Med Sci Sports Exerc. 2009;41(3):687-708.

16. de Souza Junior TP, Fleck SJ, Simão R, Dubas JP, Pereira B, de Brito Pacheco EM, et al. Comparison between constant and decreasing rest intervals: influence on maximal strength and hypertrophy. J Strength Cond Res. 2010;24(7):1843-50.

17. Baechle TR, Earle RW. Essentials of strength training and conditioning. Ed. Champaign, Human Kinetics, 2000.

18. Robertson RJ, Goss FL, Rutkowski, J, Lenz, B, Dixon, C, Timmer, et al. Concurrent validation of the omni perceived exertion scale for resistance exercise. Med Sci Sports Exerc. 2003;35(2):333-41.

19. Rhea MR. Determining the magnitude of treatment effects in strength training research through the use of the effect size statistic. J Strength Cond Res. 2004;18(4):918-20.

20. Schoenfeld BJ. Potential mechanisms for a role of metabolic stress in hypertrophic adaptations to resistance training. Sports Med. 2013;43(3):179-94.
21. Goto K, Nagasawa M, Yanagisawa O, Kizuka T, Ishii N, Takamatsu K. Muscular adaptations to combinations of highand low-intensity resistance exercises. J Strength Cond Res. 2004;18(4):730-7.

22. Schoenfeld B. The use of specialized training techniques to maximize muscle hypertrophy. Strength Cond J. 2011;33(4):60-5.

23. Hortobágyi T, Richardson SP, Lomarev M, Shamim E, Meunier $\mathrm{S}$, Russman $\mathrm{H}$, et al. Interhemispheric plasticity in humans. Med Sci Sports Exerc. 2001;43(7):1188-99.

24. Muellbacher W, Facchini S, Boroojerdi B, Hallett M. Changes in motor cortex excitability during ipsilateral hand muscle activation in humans. Clin Neurophysiol. 2000;111(2):344-9.

25. Carroll TJ, Herbert RD, Munn J, Lee M, Gandevia SC. Contralateral effects of unilateral strength training: evidence and possible mechanisms. J Appl Physiol. 2006;101(5):1514-22.

26. Carson RG, Riek S, Mackey DC, Meichenbaum DP, Willms K, Forner, M, et al. Excitability changes in human forearm corticospinal projections and spinal reflex pathways during rhythmic voluntary movement of the opposite limb. J Physiol. 2004;560(Pt 3):929-40.

27. Hortobágyi T, Taylor JL, Petersen NT, Russell G, Gandevia, SC. Changes in segmental and motor cortical output with contralateral muscle contractions and altered sensory inputs in humans. J Neurophysiol. 2003;90(4):2451-59.

28. Kawakita H, Kameyama O, Ogawa R, Hayes KC, Wolfe DL, Allatt RD. . Reinforcement of motor evoked potentials by remote muscle contraction. J Electromyogr Kinesiol. 1991;1(2):96-106.

\section{Corresponding author}

Cauê Vazquez La Scala Teixeira.

Praça Engenheiro José Rebouças, S/N, Santos, São Paulo, Brasil, 11030-000.

Email:contato@caueteixeira.com.br

Manuscript received on February 15, 2017

Manuscript accepted on August 29, 2017

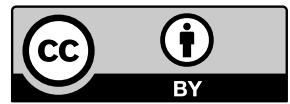

Motriz. The Journal of Physical Education. UNESP. Rio Claro, SP, Brazil - eISSN: 1980-6574 - under a license Creative Commons - Version 3.0 